\title{
Auditory Processing of Spectral Cues for Sound Localization in the Inferior Colliculus
}

\author{
Kevin A. Davis, ${ }^{1}$ Ramnarayan Ramachandran, ${ }^{1}$ and Bradford J. May ${ }^{2}$ \\ ${ }^{1}$ Department of Biomedical Engineering, Johns Hopkins University, Baltimore, MD 21205, USA \\ ${ }^{2}$ Department of Otolaryngology-HNS, Johns Hopkins University, Baltimore, MD 21205, USA
}

Received: 3 January 2001; Accepted: 29 August 2002; Online publication: 4 December 2002

\begin{abstract}
The head-related transfer function (HRTF) of the cat adds directionally dependent energy minima to the amplitude spectrum of complex sounds. These spectral notches are a principal cue for the localization of sound source elevation. Physiological evidence suggests that the dorsal cochlear nucleus (DCN) plays a critical role in the brainstem processing of this directional feature. Type $\mathrm{O}$ units in the central nucleus of the inferior colliculus (ICC) are a primary target of ascending DCN projections and, therefore, may represent midbrain specializations for the auditory processing of spectral cues for sound localization. Behavioral studies confirm a loss of sound orientation accuracy when DCN projections to the inferior colliculus are surgically lesioned. This study used simple analogs of HRTF notches to characterize single-unit response patterns in the ICC of decerebrate cats that may contribute to the directional sensitivity of the brain's spectral processing pathways. Manipulations of notch frequency and bandwidth demonstrated frequency-specific excitatory responses that have the capacity to encode HRTF-based cues for sound source location. These response patterns were limited to type $\mathrm{O}$ units in the ICC and have not been observed for the projection neurons of the DCN. The unique spectral integration properties of type $\mathrm{O}$ units suggest that DCN influences are transformed into a more selective representation of sound source location by a local convergence of wideband excitatory and frequency-tuned inhibitory inputs.
\end{abstract}

Correspondence to: Bradford J. May $\cdot$ Department of OtolaryngologyHNS • Johns Hopkins University • 720 Rutland Avenue 505 Traylor Research Building - Baltimore, MD 21205. Telephone: (410) 9553162; fax: (410) 955-1299; email: bmay@bme.jhu.edu
Keywords: sound localization, head-related transfer function, dorsal cochlear nucleus, central nucleus, spectral integration

\section{INTRODUCTION}

The filter function that describes the transformation of a free-field sound to the effective energy spectrum at the eardrum is known as the head-related transfer function (HRTF: Wightman and Kistler 1989). The properties of the HRTF are modified by changes in sound source direction and give rise to spectral cues for sound localization. In particular, the HRTF of the cat introduces a directionally dependent spectral notch at frequencies between 5 and $20 \mathrm{kHz}$ (Musicant et al. 1990; Rice et al. 1992). A special significance has been proposed for this HRTF feature because cats exhibit poorly directed sound orientation behaviors when midfrequency spectral cues are removed from free-field sounds (Huang and May 1996a).

Electrophysiological studies in cats have shown that the projection neurons of the dorsal cochlear nucleus (DCN) are especially sensitive to spectral notches (Young et al. 1992; Imig et al. 2000). When tested with pure tones, DCN principal cells exhibit a type IV frequency response map that is characterized by inhibitory responses at most combinations of frequency and level. Despite the predominance of inhibitory responses to narrowband sounds, type IV units give excitatory responses to broadband sounds. Inhibition is restored by adding a notch to the broadband spectrum near best frequency (BF: a neuron's most sensitive frequency). These nonlinear 
spectral integration properties create an unusual sensitivity to the presence of narrowband energy peaks and notches and, therefore, may serve as an early stage in the auditory processing of HRTF-based sound localization cues. Consistent with this interpretation, lesioning the output pathway of the DCN disrupts the sound orientation behaviors of cats (Sutherland et al. 1998; May 2000).

Neurons in the central nucleus of the inferior colliculus (ICC) also exhibit directional sensitivity when tested with spectrally rich sounds (Aitkin and Martin 1987, 1990; Delgutte et al. 1999). Whereas some neurons require binaural stimulation to achieve spatial selectivity, other neurons exhibit this property under monaural conditions. The presence of monaural directional sensitivity in the inferior colliculus suggests a representation of HRTF-based spectral cues that is established in the DCN and projected to the midbrain.

Functional links between the auditory brainstem and the inferior colliculus are implied by the existence of stereotypic response types in the ICC of decerebrate cats (Ramachandran et al. 1999). Each response type is presumed to reflect a discrete information processing pathway that is created under normal conditions by the convergence of ascending brainstem inputs, local processing effects, and descending efferent projections. The decerebration procedure "unmasks" the ascending inputs to these parallel processing pathways by eliminating the effects of anesthesia and removing descending influences.

Among the three major response types in the ICC of decerebrate cats, only type $\mathrm{O}$ units produce frequency response maps that share the definitive inhibitory characteristics of type IV units in the DCN (Ramachandran et al. 1999). Most type O units also fail to manifest binaural interactions that are conveyed to the ICC by non-DCN projections from the superior olive (Davis et al. 1999; Ramachandran et al. 2000; Ramachandran and May 2002). The DCN-like properties of type $\mathrm{O}$ units are silenced by applying lidocaine to the output pathways of the DCN (Davis 2002) but withstand local inhibitory blockade (Davis et al. 1999; Davis 2002).

The implied functional links between type IV and type $O$ units suggest the existence of a spectral processing pathway that is established in the DCN and maintained as a discrete response type within the inferior colliculus. This hypothetical pathway was explored in our present study by comparing the spectral integration properties of the major ICC unit types to DCN response patterns. Parametric manipulations of frequency and bandwidth using simple notched-noise analogs of the cat's HRTF revealed highly selective excitatory responses that were capable of encoding
HRTF-based spectral cues for sound localization. These responses were observed only among type $\mathrm{O}$ units and cannot be explained solely in terms of DCN influences.

\section{METHODS}

Experiments were performed on adult cats (3-4 kg) with clean external ears and clear tympanic membranes. Additional results from these subjects have been published elsewhere (Ramachandran et al. 2000). All of the following surgical and recording procedures were performed under the guidelines of the Institutional Animal Care and Use Committee of Johns Hopkins University.

\section{Surgical preparation}

Prior to surgical preparation, cats were given atropine (0.1 mg, IM) to control respiratory secretions, tranquilized with xylazine $(0.1 \mathrm{mg}, \mathrm{IM})$, and anesthetized with ketamine (initial dose $40 \mathrm{mg} / \mathrm{kg}$, IM; supplemental doses $15 \mathrm{mg} / \mathrm{kg}$, IV). Core body temperature was maintained at $39^{\circ} \mathrm{C}$ with a regulated heating pad. Cats were made decerebrate by opening the skull over the left parietal cortex and aspirating the brainstem between the superior colliculus and thalamus. Anesthesia was discontinued at this point in the surgical preparation.

A tracheotomy was performed to facilitate quiet breathing. Both ear canals were transected and the tympanic bullae ventilated to prevent static pressure buildup in the middle ear. The cat's head was secured in a stereotaxic apparatus with hollow ear bars that delivered closed-field acoustic stimuli. The left inferior colliculus was exposed by a dorsal approach through occipital cortex. Complete electrode access to the ICC was achieved by partial removal of the overlying tentorium. Cats were euthanized by barbiturate overdose (sodium pentobarbital, $100 \mathrm{mg} / \mathrm{kg}$ IV) after approximately $30 \mathrm{~h}$ of recording. Some subjects were immediately perfused to verify the completeness of decerebration and to allow histological location of recording sites.

\section{Acoustic stimuli}

Acoustic stimuli were produced with an electrostatic speaker and delivered to the contralateral (right) ear through a hollow ear bar. The frequency response of the sound system was measured before each experiment by inserting a probe tube microphone into a calibration bore on the ear bar. The tip of the probe tube was within $3 \mathrm{~mm}$ of the eardrum during calibration. The output of the sound system was meas- 
ured with a lock-in amplifier. Calibration data varied less than $\pm 5 \mathrm{~dB}$ at frequencies from 0.04 to $40 \mathrm{kHz}$.

All test stimuli were $200 \mathrm{~ms}$ in duration with $10 \mathrm{~ms}$ rise/fall times and a 1-s repetition period. Noise bursts were generated online with digital signal processing techniques (Nelken and Young 1997). Spectral notches were synthesized in the frequency domain and converted to time domain waveforms by taking the inverse Fourier transform of the digitally filtered noise spectrum. Analog signals were created by playing the waveforms through a 16 -bit $\mathrm{D} / \mathrm{A}$ converter at a sampling rate of $100 \mathrm{kHz}$.

\section{Recording protocol}

Single-unit activity was recorded with platinum-iridium microelectrodes that were advanced along the dorsoventral axis of the inferior colliculus with a hydraulic micromanipulator. The electrode signal was amplified $(10,000-30,000 \times)$ and filtered from 0.06 to $6 \mathrm{kHz}$. When a well-isolated auditory unit was encountered along the electrode track, its action potentials were discriminated from background noise using a Schmitt trigger and recorded as spike times relative to stimulus onset. The unit's BF and threshold were determined audiovisually by sweeping the frequency and level of 50-ms search tones in the contralateral ear. The electrode's entry into the central nucleus was indicated by a reversal in the trend of unit BFs relative to responses in the dorsal subdivisions of the inferior colliculus (Merzenich and Reid 1974; Aitkin et al. 1975) and by the presence of unit types that are associated with the ICC.

Each neuron was assigned to one of three major ICC unit types (V, I, and O) based on inhibitory response patterns that were characterized with contralateral tone presentations (Ramachandran et al. 1999). Initial tests involved collecting rate-level functions for BF tones and broadband noise bursts (100$\mathrm{dB}$ range in 1-dB increments). Then a frequency response map was created by sweeping the frequency of tone bursts $( \pm 2$ octaves about $\mathrm{BF})$ at multiple sound levels. Sound-driven activity was analyzed in terms of average discharge rates beginning $10 \mathrm{~ms}$ after stimulus onset to minimize adaptation effects.

The unit's sensitivity to the spectral characteristics of notched-noise was measured with two parametric procedures. A notch frequency sweep varied the center frequency of the notch from 1 octave below to 1 octave above BF. A notch rate-level series varied the level of a fixed frequency notch across the dynamic range of the unit. The notch was centered on BF and the effects of notch bandwidth were investigated by doubling the width of the notch until results were obtained at bandwidths as narrow as $200 \mathrm{~Hz}$ and as wide as 2 octaves relative to $\mathrm{BF}$.
Effects of local inhibitory inputs on the notch sensitivity of type $\mathrm{O}$ units were investigated by infusing the recording site with the $\mathrm{GABA}_{\mathrm{A}}$ antagonist bicuculline. "Piggyback' multibarreled electrodes (Havey and Caspary 1980) were made by attaching a three-barrel glass micropipette behind the tip of the metal recording electrode. Two barrels of the pipette were filled with bicuculline methiodide $(10 \mathrm{mM}, \mathrm{pH}$ 3.5-4.0, Sigma). The third barrel was filled with the $\mathrm{pH}$ balancing buffer potassium hydrogen phthalate ( $\mathrm{pH}$ 4.0, CMS). Electrode negative retention currents of $20 \mathrm{nA}$ and ejection currents of $50 \mathrm{nA}$ were produced with microiontophoresis constant current generators. Rate-level series for BF tones and broadband noise bursts were performed before and after pharmacological manipulations to verify unit stability and to confirm complete recovery from inhibitory blockade before moving on to the next unit.

\section{RESULTS}

This report is based on the responses of 94 ICC units (74 type $\mathrm{O}, 4$ type $\mathrm{V}$, and 16 type I units) that were recorded in 22 cats. A minority of high-rate type $\mathrm{O}$ units $(N=11)$ were identified by maximum tonedriven rates above 60 spikes/s and analyzed as a separate response class. The parcellation of high-rate type $\mathrm{O}$ units is based on their departure from the more generalized response patterns that link low-rate type $\mathrm{O}$ units to DCN inputs. The less common highrate type $\mathrm{O}$ units tend to be relatively insensitive to pharmacological manipulations of the dorsal acoustic stria and may exhibit radical changes in the inhibitory features of their frequency response maps during local application of bicuculline (Davis 2002). Comparison data from 20 DCN type IV units (including archival data from Nelken and Young 1994) were acquired in similar decerebrate preparations to evaluate transformations in the representation of spectral notches between the DCN and ICC.

\section{Notch sensitivity of type $\mathrm{O}$ units}

Our hypothesis that type $\mathrm{O}$ units would show specializations for the processing of spectral notches is based on the assumption that neurons in this response class receive ascending projections from the DCN (Davis 2002). A direct DCN/ICG spectral processing pathway is suggested by similarities in the frequency response maps of DCN type IV units and ICC type $\mathrm{O}$ units. Representative response maps are shown in Figures 1A and B. These results were obtained by sweeping tone frequencies across each unit's receptive field at seven SPLs. Stimulus levels are indicated in terms of $\mathrm{dB}$ attenuation to the right of 

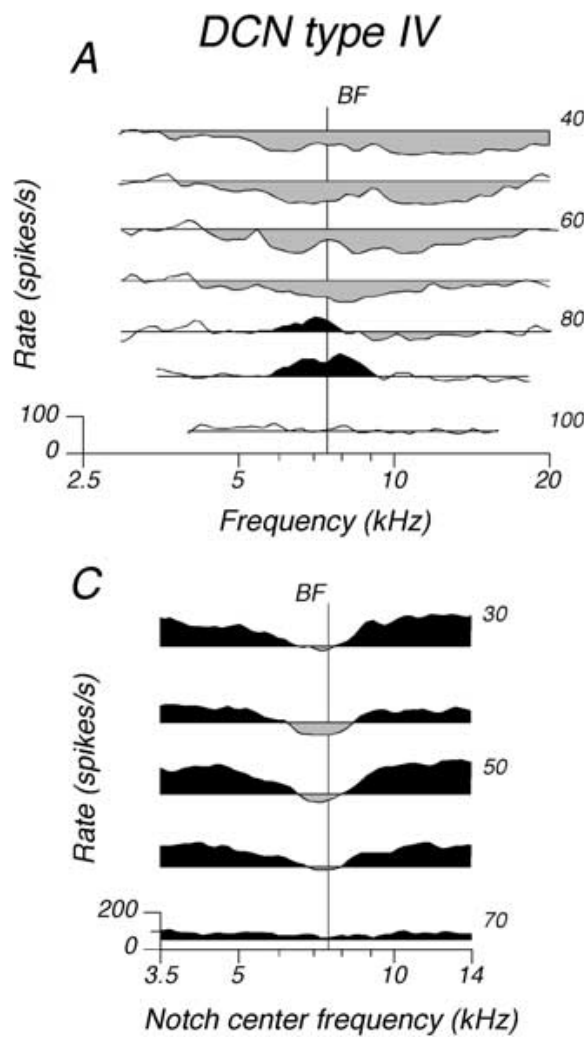

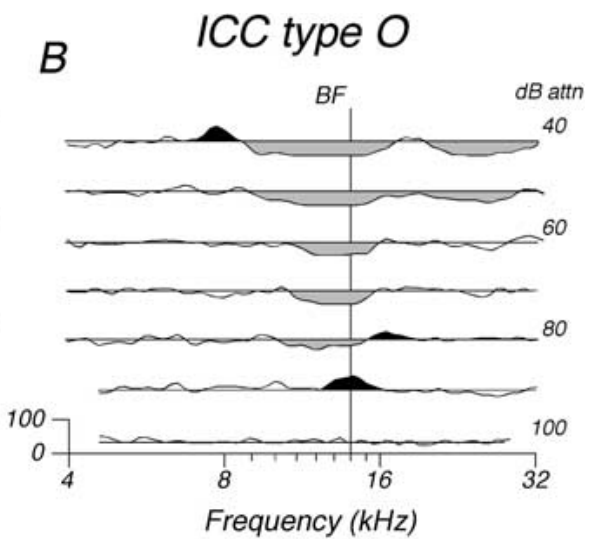

$D$

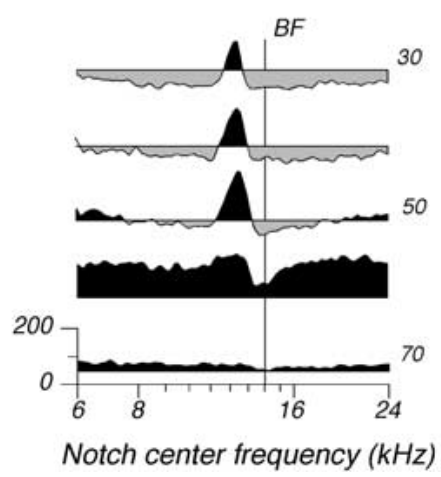

FIG. 1. Response maps for pure tones (top) and notched noise (bottom). Data were obtained from a DCN type IV unit (3.02 exp. 4/10/00, BF $=7 \mathrm{kHz}$ ) and an ICC type O unit (1.04 exp. 3/13/00, $\mathrm{BF}=14 \mathrm{kHz}$ ). The bandwidth of the notch was $3.2 \mathrm{kHz}$. The horizontal lines represent average spontaneous rates. Black (gray) regions indicate stimulus conditions that elicited excitatory (inhibitory) discharge rates. The magnitude of the rate response is indicated by the scale bar at the bottom left of each map. Stimulus levels are noted in $\mathrm{dB}$ attenuation by numerical labels along the right margin of each map. $0 \mathrm{~dB}$ attenuation is near $100 \mathrm{~dB}$ SPL re $20 \mu \mathrm{Pa}$ for tones and $40 \mathrm{~dB}$ re $20 \mu \mathrm{Pa}$ / $\sqrt{ } \mathrm{Hz}$ for noise spectrum level. each sweep. Maximum outputs (0 dB attenuation) were approximately $100 \mathrm{~dB}$ SPL across the frequency range shown here. The magnitude of the response evoked by a combination of frequency and level was measured by subtracting the unit's spontaneous rate (SR: sampled during the quiet intervals between tone presentations) from its total discharge rate during the tone presentation. The resulting driven rates are plotted relative to spontaneous rate. Excitatory response areas are defined as contiguous tone-level combinations that elicited positive driven rates and inhibitory areas are regions where tone-driven rates were less than SR.

Tone detection thresholds are defined as the lowest presentation levels that elicited driven rates at least 2 standard deviations above SR. Both of the units in Figure 1 reached the threshold criterion at a stimulus attenuation of $90 \mathrm{~dB}$. The center frequency of the excitatory response area at the threshold attenuation is BF (vertical bar).

Both units displayed frequency response maps that were dominated by inhibition except for an O-shaped island of excitation at stimulus levels near threshold and frequencies near BF. This response pattern is not observed for ICC type V or I units, which are presumed to receive their dominant ascending inputs from binaurally sensitive projection neurons in the superior olive (Ramachandran et al. 1999; Davis et al. 1999).
In spite of similarities in their pure-tone response maps, our results suggest that type $O$ and type IV units exhibit fundamental differences in their integration properties when tested with simple analogs of HRTF-based spectral notches. Representative notched-noise responses are shown in Figures $1 \mathrm{C}$ and $\mathrm{D}$. These tests were conducted by placing a single rectangular notch in flat spectrum broadband noise. The notch had a bandwidth of $3.2 \mathrm{kHz}$ and swept in frequency across a 2 octave range that was centered on BF. The notch sweep was presented at the five attenuations indicated by the numerical labels to the right of each rate profile. This range of noise levels encompassed both units' threshold responses at low levels and maximum driven rates at high levels.

The type IV unit in Figure 1C exhibited excitatory responses when the spectral notch occurred at a frequency that was remote to $\mathrm{BF}$. These positive driven rates were essentially identical to the unit's responses to broadband noise. Excitatory responses gave way to strong inhibition when the center frequency of the notch approached BF. Although the width of the tuned inhibitory response expanded slightly with increasing noise levels, strongest inhibition was observed at intermediate levels. The general properties of notch encoding in the DCN have been described in detail by previous studies (Nelken and Young 1994, 1997). 

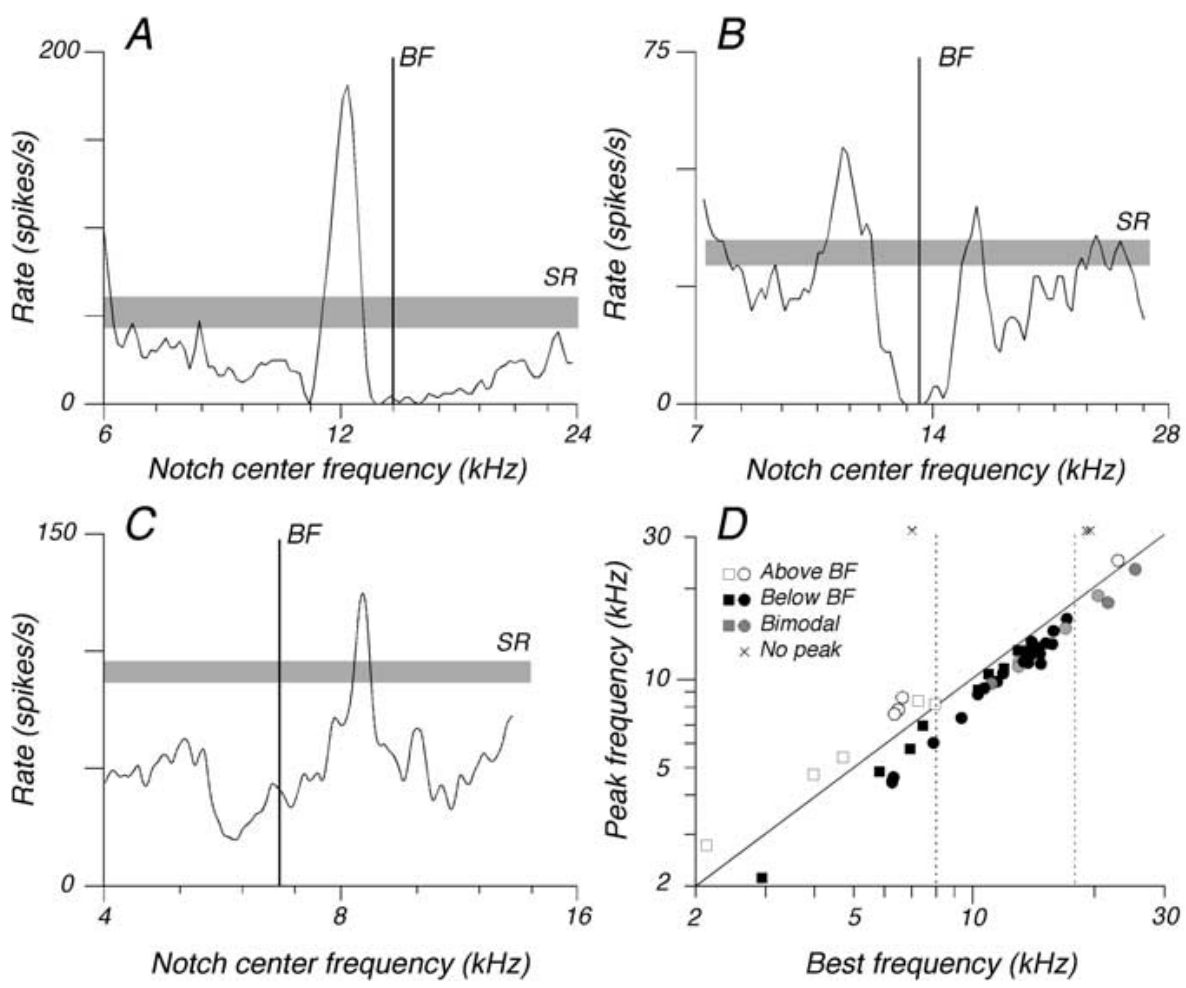

FIG. 2. Frequency distribution of notch excitatory responses. A-C. Rate profiles of type $\mathrm{O}$ units with an excitatory peak below $\mathrm{BF}$, surrounding $\mathrm{BF}$, or above BF. The gray-filled regions indicate the range of spontaneous rates (SR); vertical bars mark BF. D. Scatterplot of peak frequency versus $\mathrm{BF}$ for all type $\mathrm{O}$ units in the present study. Notch bandwidths were 1.6 (squares) or $3.2 \mathrm{kHz}$ (circles). The unity line indicates peaks that were centered on BF. Units above the ordinate did not show excitatory peaks (Xs).

The type $\mathrm{O}$ unit in Figure 1D showed notched-noise responses with almost the opposite excitatory/inhibitory characteristics of its presumed DCN inputs. These differences are most apparent at higher sound levels (50-30-dB attenuation) where the type $\mathrm{O}$ unit exhibited a tuned excitatory response that was flanked by inhibition. The excitatory peak emerged approximately $20 \mathrm{~dB}$ above threshold and was located just below BF. The magnitude of the response declined with increasing sound level but remained clearly excitatory in nature. Thus, the excitatory responses of the type $\mathrm{O}$ unit provide an unambiguous representation of notch frequency but not stimulus level.

The notch frequency selectivity of type $\mathrm{O}$ excitatory rates was evaluated in 48 units. Results from this sample are summarized in Figure 2. Of the 45 units that displayed notch sensitivity, the majority of units exhibited below-BF excitatory tuning like the unit in Figure 1D. Results from this unit have been replotted in Figure 2A to emphasize the magnitude of rate changes with notch frequency. The range of the unit's spontaneous activity during the notch sweep is indicated by the lower and upper bounds of the grayfilled region in the figure. Positive notch-driven rates are indicated by responses that exceed SR at notch frequencies near $12 \mathrm{kHz}$. This peak in the rate profile occurs below BF and corresponds to the tuned excitatory region in Figure 1D. The unit's inhibitory responses are revealed by troughs in the rate profile that dip below SR. Maximum inhibition is indicated by a complete absence of activity at frequencies im- mediately flanking the rate peak, but negative driven rates extend for almost an octave above and below the peak. Recall that DCN type IV units show strong excitation when tested with off-BF notches (Fig. 1C).

The frequency of the excitatory peak in the notch frequency function is plotted against BF for the entire sample of low-rate type $\mathrm{O}$ units in Figure 2D. Notch bandwidths are indicated by different symbol types. Symbols below the unity line in the figure indicate units that showed below-BF peaks. This response property was usually associated with units that were tuned to frequencies between 6 and $18 \mathrm{kHz}$, where spectral notches in the cat's HRTF are assumed to provide important sound localization cues (Musicant et al. 1990; Rice et al. 1992; Huang and May 1996a). The second most common response pattern was an excitatory peak above BF (Fig. 2C). These units typically had BFs below $8 \mathrm{kHz}$, as indicated by the data points that lie above the unity line in Figure 2D. Conversely, units with bimodal peaks were seen at BFs above $10 \mathrm{kHz}$ (Fig. 2B; gray-filled symbols in Fig. 2D).

The spectral integration properties of type IV and type $\mathrm{O}$ units also were investigated with notch bandwidth experiments that are described in Figure 3. A representative type IV unit displayed an on-BF tuned inhibitory response when tested with a notch bandwidth of $3.2 \mathrm{kHz}$. The trough in the unit's rate profile remained centered on BF but broadened in frequency when the bandwidth of the notch was increased to 12.8 $\mathrm{kHz}$. Clear inhibitory responses were observed only at the edges of the trough during tests with wideband 

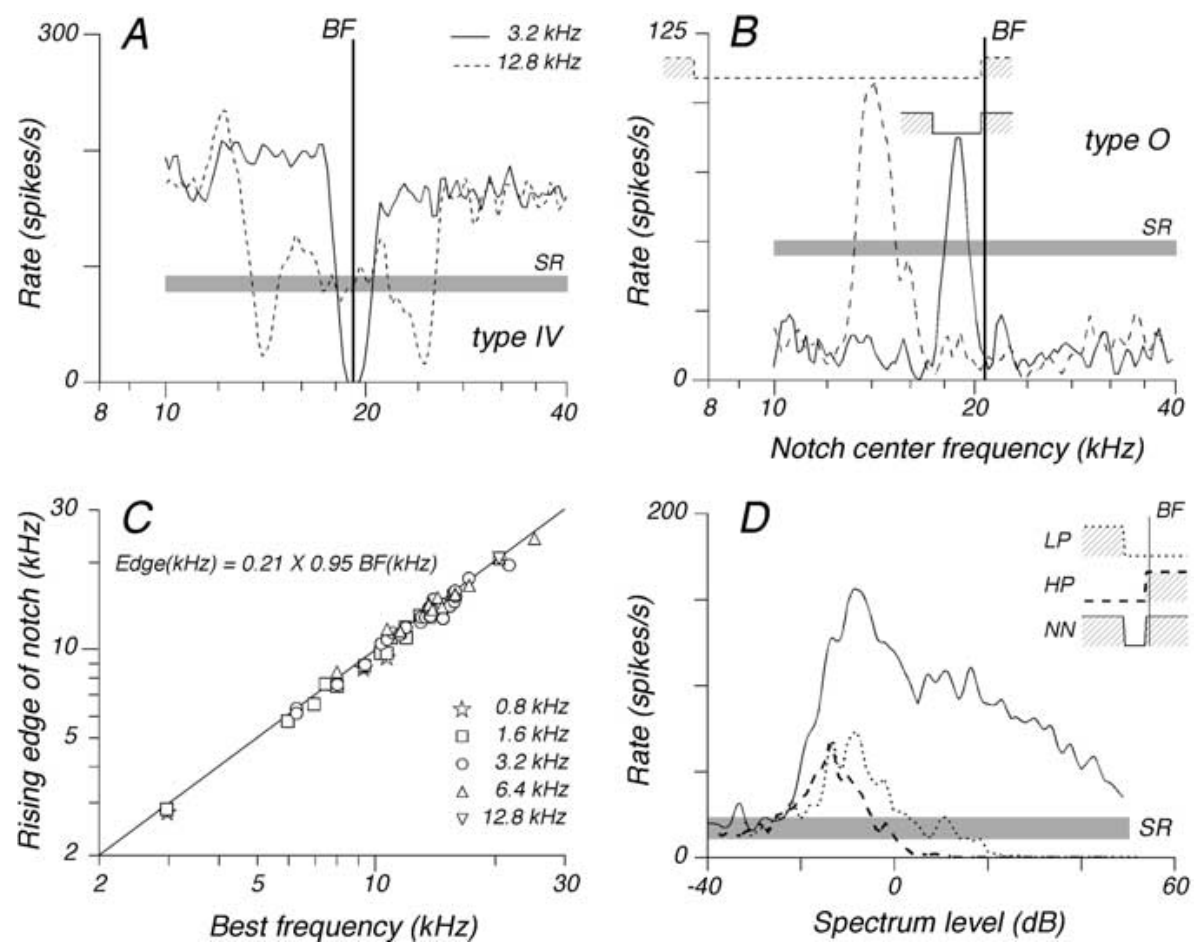

FIG. 3. Interactions of notch frequency and bandwidth on the excitatory responses of type $\mathrm{O}$ units. A, B. For comparison, data are shown for a representative type IV unit(4.01 exp. 9/ $5 / 00, \mathrm{BF}=19 \mathrm{kHz})$ and type $\mathrm{O}$ unit (1.06 exp. 6/24/99, BF $=21 \mathrm{kHz}$ ). Insets show the location of spectral edges for notches that elicited maximum excitatory responses from the type $\mathrm{O}$ unit. C. Scatterplot of the rising edge of excitatory notches versus BF for type $\mathrm{O}$ units with below-BF notch sensitivity. Symbols identify different notch bandwidths. The equation describes a statistically significant linear fit to the data. A unity line is superimposed to emphasize the slight negative displacement of edge frequency relative to BF. D. Responses of a type $\mathrm{O}$ unit (2.05 exp. $3 / 13 / 00, B F=11 \mathrm{kHz}$ ) to spectral edges versus spectral notches. As illustrated by the inset, stimulus conditions were low-pass noise (LP, dotted line), high-pass noise (HP, dashed line), and notched noise that represented the sum of the bandpass stimuli (NN, solid line). notches. By contrast, a representative type $\mathrm{O}$ unit exhibited excitatory responses that were limited to a narrow range of frequencies below BF. This unit maintained its tuned excitation when notch bandwidth was progressively increased from 3.2 to $12.8 \mathrm{kHz}$, but the rate peak shifted to lower frequencies. The downward displacement of the rate peak implies the type $\mathrm{O}$ unit may have been responding to the highpass or rising edge of the notch (insets).

The frequency of the rising edge of the notch is plotted as a function of BF in Figure 3C. Results are shown for all type $\mathrm{O}$ units with response peaks below $\mathrm{BF}$ and are limited to the peak frequency at each bandwidth. Most units were tested at several bandwidths and, therefore, contributed multiple data points to the figure.

The statistically significant linear relationship of notch edge to $\mathrm{BF}$ is summarized by the regression equation in the figure $(R=0.99, p<0.001)$. The slope of the regression showed a small but significant deviation from unity, indicating the negative displacement of the rising edge of the spectral notch slightly increased in magnitude at higher BFs. The relationship was not influenced by notch bandwidth. Regression analyses were not performed on the sparsely sampled type $\mathrm{O}$ units with excitatory peaks above BF or bimodal peaks.

The correlation between the rising edge of spectral notches and the frequency location of excitatory response areas suggests that type $\mathrm{O}$ units may derive their sensitivity to directional features of the HRTF by processing spectral edges and not notches, per se. This possibility was explored by measuring the responses of type $\mathrm{O}$ units $(N=5)$ to the component elements of spectral notches. Three types of bandlimited noise were compared. The notch condition had a fixed bandwidth of $1.6 \mathrm{kHz}$ and was centered on the frequency that evoked the unit's maximum excitatory response during tests with notch frequency sweeps. The low-pass noise condition had an upper cutoff frequency at the falling edge of the notch. The highpass noise condition matched the rising edge of the notch. Thus, the notched-noise spectrum was equivalent to the sum of the low- and high-pass stimuli.

Representative rate-level functions for notch component experiments are shown in Figure 3D. The matched cutoff frequencies of the three stimulus conditions are schematized in the inset of Figure 3. When tested with either high-pass or low-pass noise, the type $\mathrm{O}$ unit attained peak discharge rates approaching 75 spikes/s and showed strong inhibitory responses at high sound levels. The unit responded to notched noise with discharge rates exceeding 150 spikes/s and maintained excitatory responses at high sound levels. A selective response to notches, and not spectral edges, was observed in all sampled type $O$ units $(5 / 5$ cases $)$.

\section{Notch sensitivity of other unit types}

The notch sensitivity of the ICC unit types that show less direct links to the DCN are summarized in Fig- 

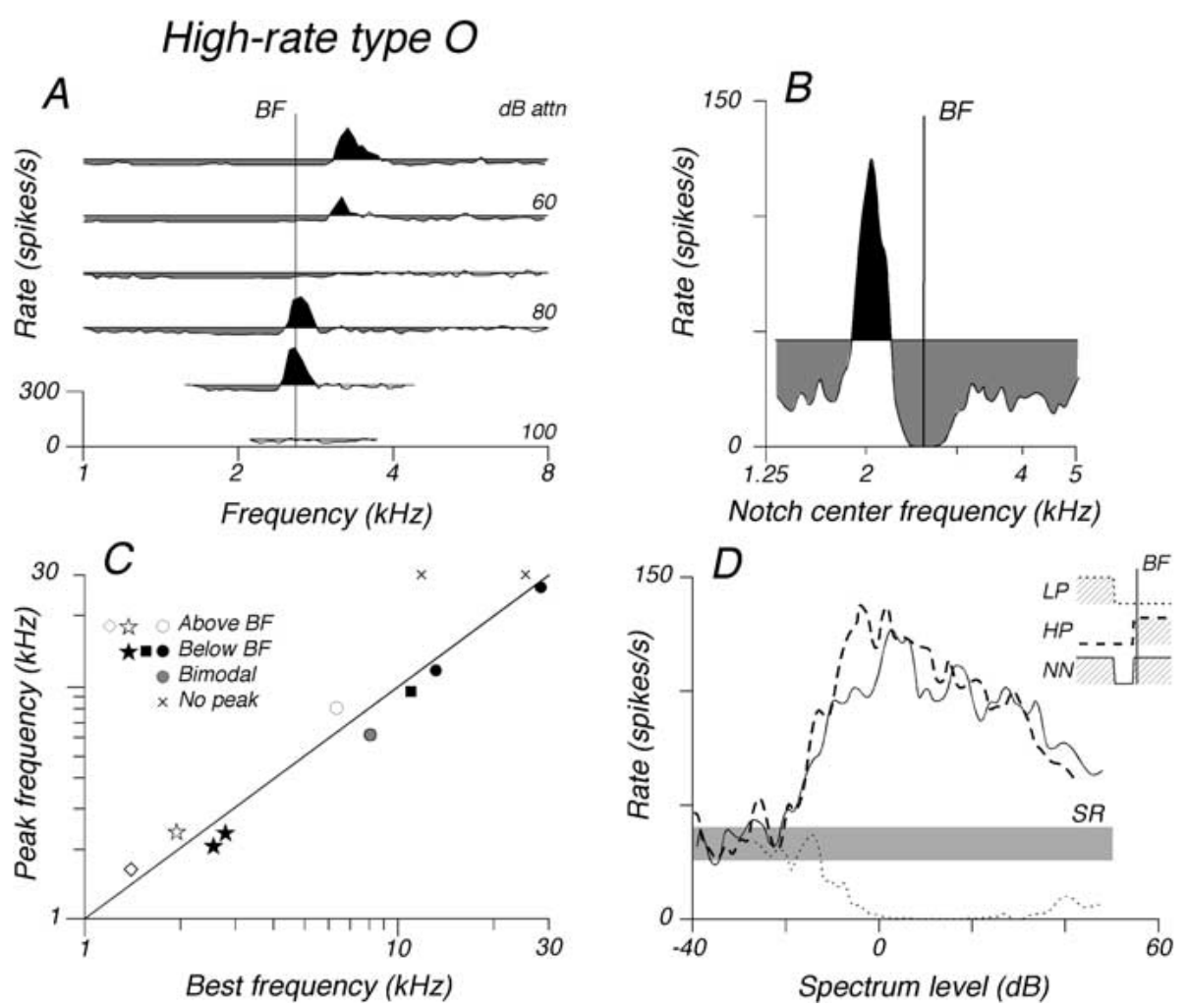

FIG. 4. Response patterns of high-rate type $\mathrm{O}$ units. A, B. Pure-tone response map and notched noise rate profile for a representative high-rate type $\mathrm{O}$ unit (4.01 exp. 9/12/00). C. Scatterplot of peak frequency versus BF for all sampled high-rate type $\mathrm{O}$ units. Different symbols indicate different notch bandwidths: diamonds, $0.4 \mathrm{kHz}$; stars, $0.8 \mathrm{kHz}$; squares, $1.6 \mathrm{kHz}$; and circles, $3.2 \mathrm{kHz}$. The diagonal line indicates peaks that were centered on BF. D. Responses of the type $\mathrm{O}$ unit to spectral edges versus spectral notches. The inset shows the complementary frequency arrangement of the three stimulus conditions. ures 4 and 5 . These units include the less common high-rate type $\mathrm{O}$ units, MSO-like type $\mathrm{V}$ units, and LSO-like type I units.

In general, the high-rate type $\mathrm{O}$ units produced frequency response maps and notched-noise response patterns that captured the essential properties of low-rate type $\mathrm{O}$ units (Figs. 4A and 4B). The highrate type $\mathrm{O}$ units exhibited tuned excitatory responses to notch frequency sweeps which in most instances produced a selective response to notch frequencies below BF (Fig. 4C). The remaining highrate type $\mathrm{O}$ units exhibited above-BF or bimodal excitatory tuning. The proximity of the high-pass (or low-pass) edge of the notch to the unit's BF elicited the excitatory peak, regardless of the bandwidth of the notch or the BF of the unit.

High-rate type $\mathrm{O}$ units failed to display notch-selective responses that were typical for low-rate type $\mathrm{O}$ units. These differences were revealed by the inhibitory properties of rate-level functions for low-pass, high-pass, and notched noise. For example, the representative high-rate type $\mathrm{O}$ unit exhibited nearly identical excitatory discharge rates when tested with high-pass or notched noise (Fig. 4D). This added sensitivity to spectral edges suggests the unit's spectral coding is less selective than the notch-driven activity of low-rate type $\mathrm{O}$ units. Similar patterns were observed for all sampled high-rate type $\mathrm{O}$ units $(4 / 4$ cases).
Type V units in the ICC of decerebrate cats are identified by $\mathrm{V}$-shaped frequency response maps that are exclusively excitatory (Fig. 5A). Type V units also display low frequency tuning (Ramachandran et al. 1999) and peak-type interaural time difference sensitivity (Ramachandran and May 2002). These response patterns are assumed to reflect a dominant ascending input from the MSO (Batra et al. 1997).

Type $\mathrm{V}$ units showed only excitatory responses when tested with notch frequency sweeps (Fig. 5C). A decrease in response magnitude was observed as the notch neared BF and spectral energy was removed from the unit's excitatory response area. Similar response patterns have been previously described in the auditory nerve (Poon and Brugge 1993). Since discharge rates in the MSO pathway are tightly coupled to auditory nerve inputs by powerful calyceal synapses (Cant and Casseday 1986), it is not surprising that type $\mathrm{V}$ units did not provide a significant transformation of HRTF encoding relative to peripheral representations.

The frequency response maps of type I units are distinguished by level-tolerant excitation that is bounded by lateral inhibition (Fig. 5B). When tested with notch frequency sweeps, units in this response class showed on-BF inhibitory responses that weakened with increasing sound level (Fig. 5D). Similar responses have been previously described for DCN type IV units (Fig. 1C). Both unit types provide sen- 


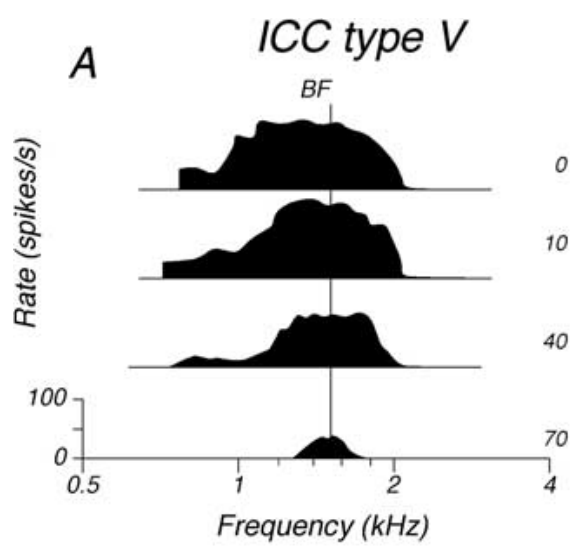

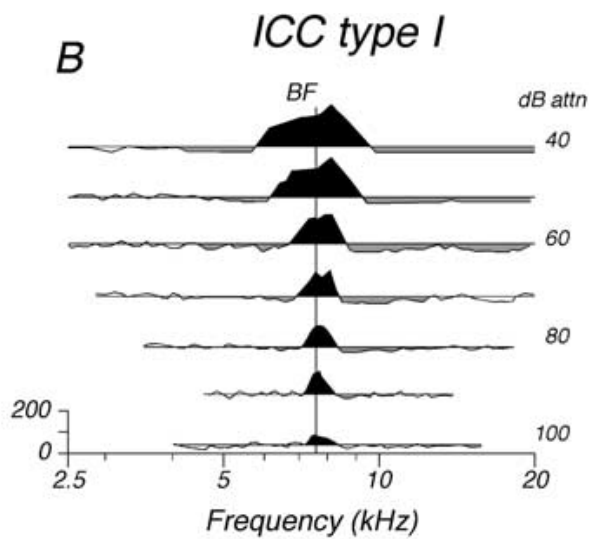

$D$

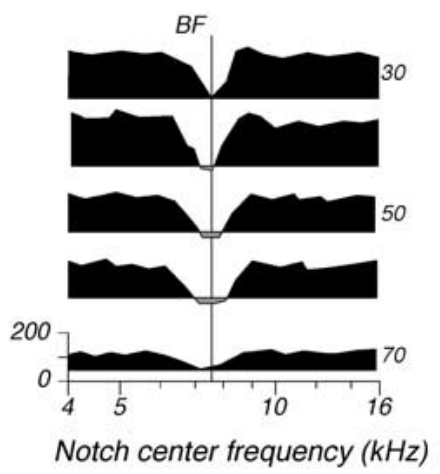

FIG. 5. Sensitivity of type $V$ and I units to notched noise. Response maps for pure tones (top) and notched noise (bottom) for a type $\mathrm{V}$ unit (2.01 exp. 3/ 13/00) and type I unit (1.02 exp. 4/20/ 00). The bandwidth of the notch was 0.4 $\mathrm{kHz}$ for the type $\mathrm{V}$ unit, and $1.6 \mathrm{kHz}$ for the type I unit. sitive but not selective responses to spectral notches because they are equally inhibited by a variety of alternate stimulus conditions including off-BF tones (Fig. 5B).

\section{Spectral integration properties of type $\mathrm{O}$ units}

Early evidence for functional links between the DCN and ICC was based on the similarity of pure-tone response maps for type IV and type $\mathrm{O}$ units. Systematic differences in the spectral integration properties of the two unit types suggest that additional inputs to the inferior colliculus may enhance the representation of spectral notches that is provided by type IV units. These differences provide insights into the physiological properties of currently unknown sources of input to type $\mathrm{O}$ units.

The integration bandwidths of type IV and type $\mathrm{O}$ units were determined by measuring the effects of notch-widening experiments on noise-driven rates. Rate-level functions are presented in Figures 6A and $\mathrm{B}$ to define methods for quantifying notch bandwidth effects. Each set of rate-level functions was obtained by sweeping the level of notched noise across the dynamic range of the unit under study. The notch was fixed in center frequency at $\mathrm{BF}$ and varied in bandwidth from 0.2 to $12.8 \mathrm{kHz}$. The type $\mathrm{O}$ unit exhibited excitatory responses at low sound levels and inhibitory responses at high levels. Increasing notch bandwidth reduced the magnitude of the unit's peak discharge rates but did not change this general response pattern. The type IV unit shifted from excitatory to inhibitory responses as notch bandwidth increased.

Statistical descriptions of the effects of notch bandwidth are based on 18 low-rate type $\mathrm{O}$ units and archival data from 15 type IV units (Nelken and Young 1994). The mean driven rates of the sample of type $\mathrm{O}$ units are plotted as a function of notch bandwidth in Figure 6C. These results were calculated by averaging individual rate-level functions like those shown in Figure 6A and are limited to responses at 10 $\mathrm{dB}$ re threshold. The results have been normalized by subtracting spontaneous activity. Consequently, excitatory and inhibitory responses are indicated by positive and negative driven rates.

Type $\mathrm{O}$ units showed excitatory responses at all notch bandwidths when tested at low sound levels. Positive driven rates declined, but were not eliminated, as the passbands of the noise moved away from $\mathrm{BF}$. These off-BF responses are presumed to reflect influences of additional excitatory inputs. It is unlikely that this excitation was produced by an on-BF type IV input since type IV units were strongly inhibited at increasing notch bandwidths. Analyses of the effects of notch bandwidth on rate-level functions for our entire sample of units indicated the median excitatory bandwidth extended across 1.0 octave for 

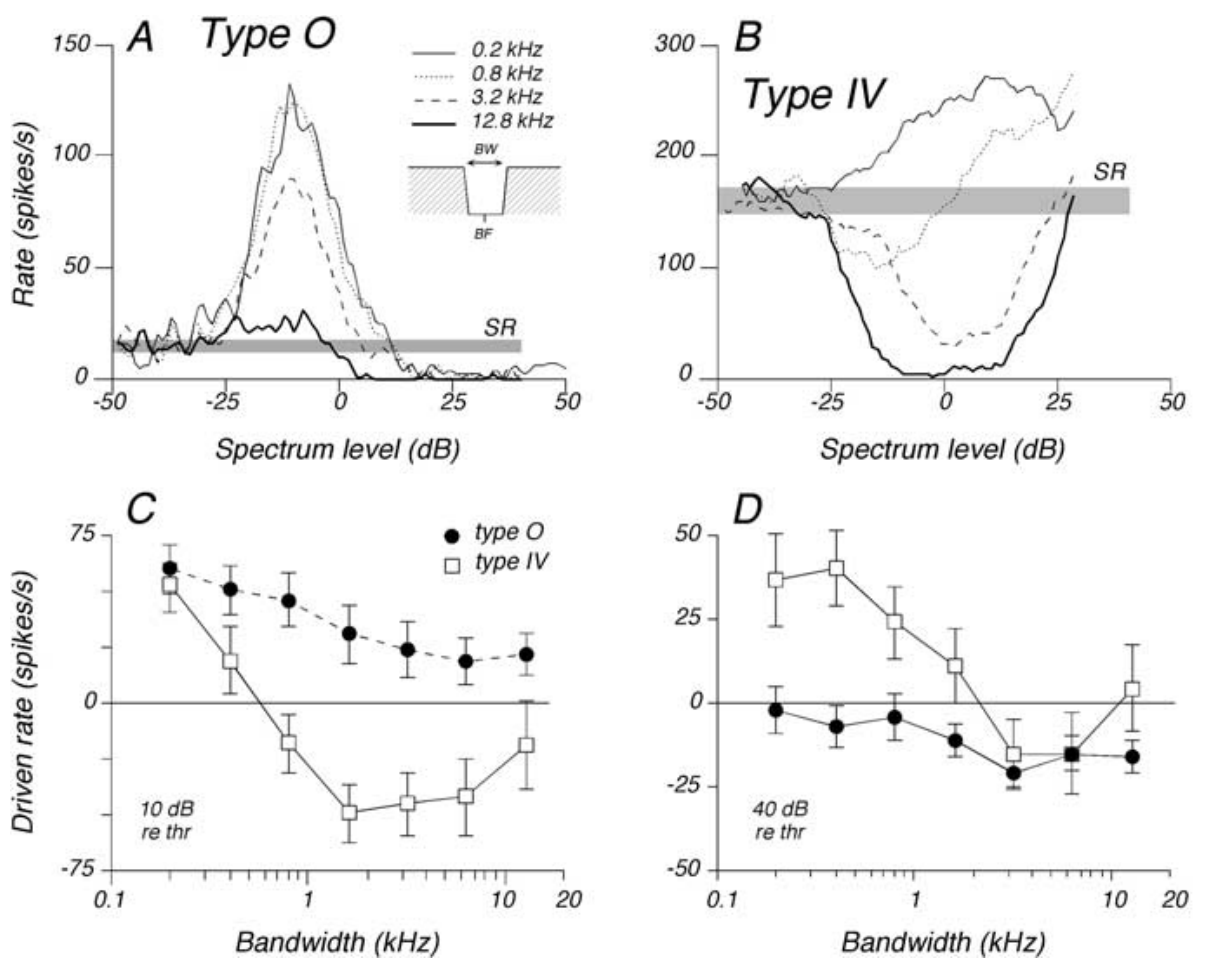

FIG. 6. Effects of notch bandwidth on the responses of type IV and type $\mathrm{O}$ units. A, B. Rate-level functions for a type $\mathrm{O}$ unit (1.12 exp. 4/30/97, BF $=23$ $\mathrm{kHz})$ and type IV unit (1.04 exp. 3/18/ 93, $\mathrm{BF}=15 \mathrm{kHz}$ ) at four notch bandwidths (see legend in A). C. Effects of bandwidth on the average driven rates of type $\mathrm{O}$ units (filled circles) and type $\mathrm{O}$ units (squares) at a noise level $10 \mathrm{~dB}$ re threshold. D. Average driven rates at a $40-\mathrm{dB}$ noise level. Data for type IV units are taken from Nelken and Young (1994).

type $\mathrm{O}$ units and was limited to only 0.14 octave for type IV units. The sevenfold difference in the spectral integration patterns of the two unit types was statistically significant ( $p=0.01$, Student's $t$ test).

The effects of notch bandwidth at high sound levels are summarized in Figure 6D. This analysis is limited to responses at $40 \mathrm{~dB}$ re threshold. Type $\mathrm{O}$ units were largely inhibited under the more intense stimulus condition, particularly at wider notch bandwidths. This shift toward more complete inhibition may reflect a concomitant decrease in opposing excitatory influences, which is most obvious at low sound levels (Fig. 6C). Nevertheless, additional inhibitory inputs are required to account for differences in the response patterns of type IV and type $\mathrm{O}$ units at narrow notch bandwidths and high sound levels.

\section{Pharmacological evidence for local inhibitory inputs}

Bicuculline was applied to recording sites in the ICC to assess how GABAergic inhibitory inputs influenced the notch frequency selectivity of three type $\mathrm{O}$ units. The results of these pharmacological manipulations are summarized by representative responses to notch frequency sweeps in Figure 7. Prior to drug administration, the type $\mathrm{O}$ unit showed strictly excitatory responses at low sound levels (10 $\mathrm{dB}$ re threshold). The magnitude of the response fell sharply, but was not inhibited below levels of spontaneous activity, as the notch swept across BF. Inhibitory effects dominated the unit's responses at high presentation levels (40 $\mathrm{dB}$ re threshold). As a result, the range of excitatory notch frequencies contracted to a narrow peak below $\mathrm{BF}$. These results replicate the general notch-selective responses of type $\mathrm{O}$ units (Fig. 1D).

The most obvious effect of bicuculline was to raise the responsivity and lower the selectivity of the type $\mathrm{O}$ unit's responses to notched noise. In addition to higher rates of spontaneous activity, noise-driven rates increased at all off-BF notch frequencies. Notwithstanding this robust increase in response magnitude, general trends in the unit's responses to notch sweeps were preserved at low sound levels.

Substantial changes were noted at high sound levels where inhibition is expected to play an important role in shaping notch selectivity (Fig. 6D). Most notably, the unit's excitatory response area expanded to include remote notch frequencies that previously evoked strong inhibition creating an on-BF inhibitory trough that was flanked by broadband excitation. This response pattern is typical of type IV units (Fig. 1C). Bicuculline administration also transformed the inhibitory effects of broadband noise and narrow notch bandwidths to type IV-like excitatory responses. The persistence of type IV response patterns in the presence of these pharmacological manipulations represents another line of evidence supporting the existence of direct DCN inputs to type $\mathrm{O}$ units in the inferior colliculus. 
The frequency tuning characteristics of local GABAergic inputs to the type $\mathrm{O}$ unit also can be inferred from the effects of bicuculline. Of particular interest is the observation that the unit exhibited strictly excitatory responses to notch frequencies below BF during drug administration. This loss of selectivity suggests that a tuned excitatory peak occurs in the notched-noise responses of type $\mathrm{O}$ units because local inhibitory inputs are active at all but a narrow range of near-BF notch frequencies. Variations in preferred notch frequencies are likely to reflect the tuning of the inhibition. Results in Figure 2D suggest this tuning may be biased to enhance the processing of naturally occurring spectral notches. That is, above-BF notch sensitivity is usually observed at BFs below the normal range of midfrequency notches in the cat's HRTF. Bimodal sensitivity is associated with higher BFs where the HRTF exhibits more complex filtering effects.

\section{DISCUSSION}

Our major findings support the hypothesis that DCN type IV units and ICC type $\mathrm{O}$ units contribute to an auditory pathway that is uniquely specialized for processing directionally dependent spectral features of the cat's HRTF. Within the inferior colliculus, ascending projections from the DCN are transformed by the combined effects of local excitatory and inhibitory inputs to enhance the selective encoding of spectral notches and, therefore, sound location. The following discussion summarizes these results in terms of a simple connectionist model and evaluates candidate neurons that may serve as currently unknown sources of input to type $\mathrm{O}$ units. Then the functional implications of the spectral processing pathway are interpreted in the context of the cat's sound localization behaviors. Finally, issues regarding the generalization of results from decerebrate cats to other species are addressed.

\section{A connection model of the type $\mathrm{O}$ unit}

A hypothetical circuit diagram of the prototypic $\mathrm{O}$ unit is shown in Figure 8. Results that have led to this connection model are summarized in Table 1, which compares DCN type IV and ICC type $\mathrm{O}$ response patterns across critical stimulus parameters.

Shared properties of type IV and type O units are presumed to indicate a direct on-BF excitatory input from the type IV unit (designated input IV in Fig. 8). Our previous studies have shown that both unit types are excited by BF tones at low sound levels and inhibited at high levels (Table 1). Consequently, the excitatory island that defines the frequency response
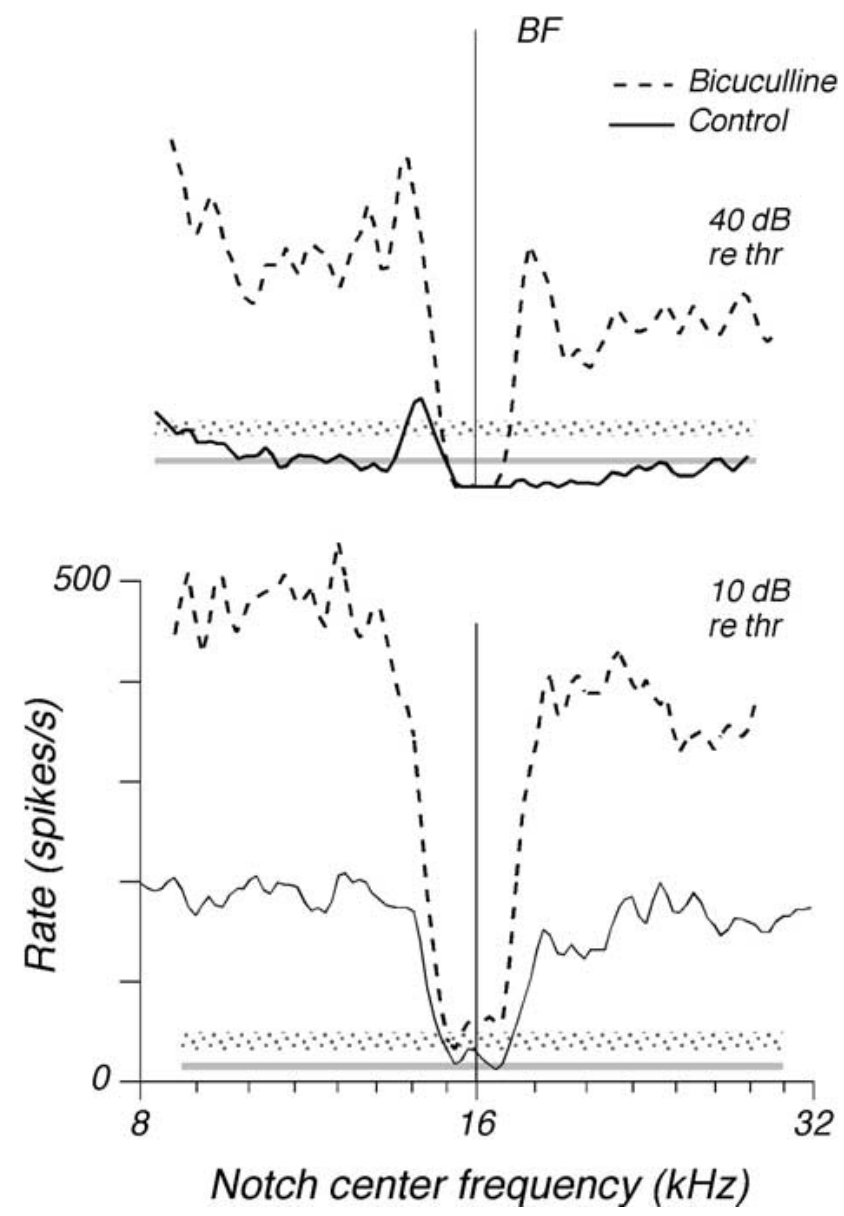

FIG. 7. Effects of bicuculline on the responses of a type $\mathrm{O}$ unit (2.18 exp. 7/12/99) to notch frequency sweeps. Discharge rates were recorded before (solid lines) and during (dashed lines) bicuculline administration at noise levels 10 and $40 \mathrm{~dB}$ above threshold. Ranges of spontaneous rates before and during drug application are indicated by the width of gray and stippled bars, respectively.

map of a type IV unit in the DCN is conserved by a type $\mathrm{O}$ unit in the ICC (Figs. 1A and B).

The responses of type IV and type $\mathrm{O}$ units diverge when spectral integration patterns are measured with complex sounds. Stimulus conditions where type IV excitation shifts to type $O$ inhibition suggest an additional inhibitory input (designated INH in Fig. 8 and Table 1). For example, broadband noise elicits excitatory responses from type IV units at high sound levels (Young and Brownell 1979; Young et al. 1992). Type $\mathrm{O}$ units are inhibited under the same stimulus conditions (Ramachandran et al. 1999).

The results of notch frequency manipulations suggest that INH influences are tuned in frequency. At high sound levels, type IV responses change from inhibition to excitation as the spectral notch sweeps from BF to remote frequencies (Fig. 1C). By contrast, type $\mathrm{O}$ responses are strongly inhibited except when the notch removes energy from a narrow band of frequencies near BF (Fig. 1D). This selective excita- 


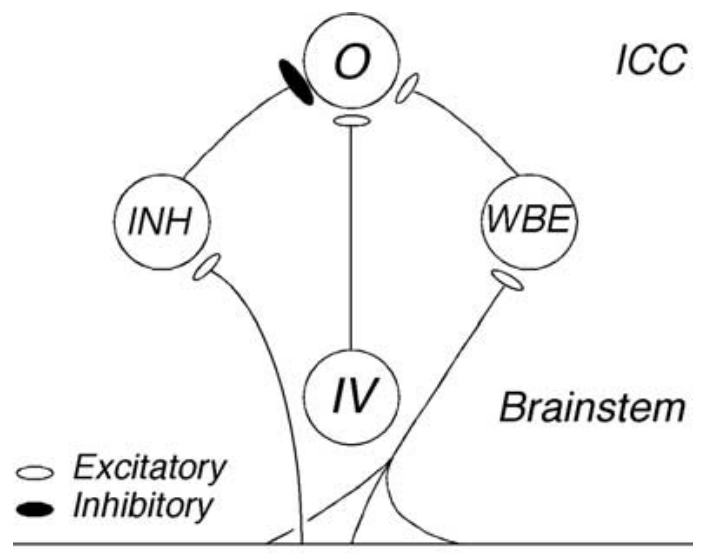

Best frequency of input

FIG. 8. Circuit diagram summarizing hypothetical sources of input to a prototypic $\mathrm{O}$ unit. The tonotopic organization of excitatory (filled symbols) and inhibitory inputs (open symbols) are indicated at the bottom of the figure. IV: On-BF excitatory input from a DCN type IV unit. INH: Inhibitory input with below-BF frequency tuning. WBE: Excitatory input with wideband spectral integration properties.

tion presumably occurs because the INH input has the capacity to overwhelm the unit's excitatory inputs but requires energy in the frequency range that is revealed by the excitatory peak of the notch frequency function.

Type IV inhibitory responses fail to predict type $\mathrm{O}$ excitatory responses when on-BF spectral notches are presented at low sound levels (Figs. 1C and D). This transformation makes it necessary to add an excitatory input to the prototypic O unit (designated WBE in Fig. 8 and Table 1). The integration properties of this so-called "wideband exciter" are inferred from its ability to influence type $\mathrm{O}$ responses at very broad notch bandwidths (Fig. 6C). It is assumed that the WBE input is weaker than the INH input at high sound levels where type $\mathrm{O}$ units are largely inhibited.

\section{Candidate inputs for the type $\mathrm{O}$ circuitry}

The prototypic $\mathrm{O}$ unit receives an on-BF excitatory input from a type IV unit. Anatomical evidence is well established for contralateral DCN projections to the inferior colliculus (Ryugo et al. 1981; Oliver et al. 1997). Our functional interpretations of the ICC targets of these ascending brainstem projections are based on single-unit response patterns in decerebrate cats. In the context of the decerebrate preparation, type IV and type $\mathrm{O}$ units show similar pure-tone response maps (Ramachandran et al. 1999) and binaural sensitivity (Davis et al. 1999; Ramachandran and May 2002). In addition, the activity of low-rate type $\mathrm{O}$ units ceases during pharmacological blockade of the DCN output pathways (Davis 2002).
Our model assumes that type $\mathrm{O}$ units acquire notch selectivity by the action of near-BF inhibitory inputs. The INH input is expected to arise from a direct projection to the inferior colliculus since type $\mathrm{O}$ units become more responsive to off-BF spectral notches when GABAergic inhibition is blocked by local application of bicuculline (Fig. 7, Table 1). INH effects dominate type $\mathrm{O}$ responses at high sound levels, except when a spectral notch removes energy from a narrow range of frequencies near BF (Figs. 2A-C). The INH input is available for type $\mathrm{O}$ units with BFs ranging from 2 to $30 \mathrm{kHz}$ (Fig. 2D).

GABAergic inhibitory neurons within the ICC respond well to narrowband sounds (Oliver and Beckius 1992). Although the neurotransmitter systems of type I and type $\mathrm{V}$ units are unknown and cannot be directly linked to local GABAergic influences, these neurons are interesting candidates for the INH input. In particular, type I units display narrow frequency tuning at high sound levels and encompass a broad range of BFs. Type $\mathrm{V}$ units seem less likely to provide the INH input because they show broad tuning at high levels and exist within a restricted range of BFs (Ramachandran et al. 1999). External sources of the INH inputs include GABAergic projections from the dorsal nucleus of the lateral lemniscus (Shneiderman et al. 1993).

Our connection model assumes a source of wideband excitation to the prototypic $\mathrm{O}$ unit. The WBE input is used to explain excitatory responses to on-BF spectral notches at low sound levels (Figs. 1D). Type IV units tend to be inhibited under the same stimulus conditions (Fig. 1C). The WBE input appears to be relatively ineffective under narrowband conditions since the off-BF inhibitory properties of type $\mathrm{O}$ response maps are not fundamentally different from those of DCN type IV units (Figs. 1A and B). The broad spectral integration properties of the WBE input are revealed by the persistence of excitatory responses at notch bandwidths reaching $12.8 \mathrm{kHz}$ (Fig. $6 A)$. Type IV units are inhibited under these conditions (Fig. 6B). Although type $\mathrm{O}$ responses predict a WBE input that is about seven times wider than the excitatory bandwidths of DCN type IV units, a wideband integration could arise from convergent type IV units with interleaved excitatory response areas. DCN sources of wideband excitation are supported by the observation that type $\mathrm{O}$ units cease activity when the propagation of action potentials in the dorsal acoustic stria is blocked with lidocaine (Davis 2002).

Onset units in the ICC are a second candidate for the WBE input. Although less than 10\% of ICC neurons show onset responses in decerebrate cats (Ramachandran et al. 1999), this unit type manifests the requisite response properties of the WBE under a variety of stimulus conditions. Onset units are weakly 
TABLE 1

Spectral integration properties of DCN type IV and ICC type O units

\begin{tabular}{|c|c|c|c|c|}
\hline \multirow[b]{2}{*}{ Stimulus } & \multicolumn{2}{|c|}{ Low level } & \multicolumn{2}{|c|}{ High level } \\
\hline & $D C N I V$ & ICC O & $D C N I V$ & ICCO \\
\hline BF tone & Exc & Exc & Inh & Inh \\
\hline Broadband noise & Exc & Exc & Exc & INH (Exc) \\
\hline \multicolumn{5}{|l|}{ Notch frequency } \\
\hline On-BF & Inh & WBE & Inh & Inh \\
\hline Below-BF & Exc & Exc & Exc & Exc \\
\hline Remote & Exc & Exc & Exc & INH (Exc) \\
\hline \multicolumn{5}{|l|}{ Notch bandwidth } \\
\hline Narrow & Exc & Exc & Exc & INH (Exc) \\
\hline Wide & Inh & WBE & Inh & Inh \\
\hline
\end{tabular}

Responses in bold indicate modifications of DCN inputs by additional sources of input proposed in Fig. 8. Responses in parentheses were observed during bicuculline administration.

driven by narrowband stimuli but sustain robust excitatory responses to noise bursts. They are broadly tuned and respond well to wideband sounds with spectral notches. The excitatory response weakens with increasing sound levels, which would explain why type $\mathrm{O}$ units are dominated by INH inputs at high levels. Onset units also are sensitive to lidocaine treatments of the dorsal acoustic stria (Davis 2002). These results suggest that DCN type IV units may combine to create the WBE input either through direct projections to type $\mathrm{O}$ units or indirectly through projections to onset units.

Our parametric study of spectral coding specializations in the ICC is based on responses to the most elementary analogs of HRTF notches. Stimulus control was further enhanced by testing under monaural conditions with fixed frequencies and spectral shapes. Other investigators have shown that the perception and processing of directional information may change under more natural dynamic conditions that are not addressed by our model of type $\mathrm{O}$ inputs (e.g., Shu et al. 1993; Spitzer and Semple 1991). Unexplored binaural influences also are implied by the excellent ITD sensitivity of some type $\mathrm{O}$ units (Ramachandran and May 2002). Although currently proposed inputs cannot account for these important aspects of sound localization, the type $\mathrm{O}$ model provides a very specific context for isolating these additional sources of input in future studies.

\section{Processing and perception of spectral cues for sound localization in cats}

Behavioral paradigms have established that cats show well-directed head orientation responses to broadband sounds that originate in the frontal field (May and Huang 1996). This accurate pattern of orientation behavior is relatively unaffected when stimulus bandwidth is decreased in a manner that preserves the availability of midfrequency spectral notches (Huang and May 1996a). In contrast, poorly directed orientation responses are observed when midfrequency spectral cues are removed by high-pass filtering or by narrowing the bandwidth of the stimuli to midfrequency pure tones. Cats also show a loss of performance, particularly in their orientation to sound source elevations, when the pathway that links the DCN to the ICC is surgically lesioned (Sutherland et al. 1998; May 2000). These observations are consistent with the interpretation that the auditory processing of spectral cues for sound localization is established in the DCN by type IV units and then refined in the ICC by type $\mathrm{O}$ units.

The directional dependence of midfrequency spectral notches is illustrated in Figure 9A for three elevations in the median plane (Musicant et al. 1990; Rice et al. 1992). If sound sources are restricted to this plane of reference, the monaural processing of notch frequency is capable of providing unambiguous information for the identification of stimulus elevation. If the potential locations of sound sources are expanded to define a two-dimensional sound field, notch frequency does not represent a unique point in space but rather a contour connecting high contralateral elevations to low ipsilateral elevations. Three HRTFs that fall along an 11-kHz notch contour are illustrated in Figure 9B. The notch-selective responses of type $\mathrm{O}$ units (Fig. 1D) predict that these stimuli will produce a spatial receptive field in which excitatory discharge rates parallel the unit's preferred notch frequency contour. A similar pattern of inhibitory activity has been previously described for DCN type IV units (Imig et al. 2000).

The importance of spectral cues for sound localization can be evaluated in a more natural context by testing with virtual sound fields (VSF). These stimulus conditions allow precise specification and independent manipulation of simulated directional informa- 
tion under closed-field conditions. Our preliminary results with this procedure are presented in Figure 9C, which shows the responses of a type $\mathrm{O}$ unit to binaural VSF stimuli that conveyed HRTF-based spectrum, level, and timing information at 99 locations in the frontal field (Rice et al. 1992). The bold line in Figure 9 identifies the spatial contour where HRTFs matched the preferred notch frequency of the unit $(11 \mathrm{kHz})$. The well-defined rate peak in the unit's responses along the contour indicates much greater spatial tuning than is predicted by DCN response patterns or type $\mathrm{O}$ notch selectivity.

The HRTF of the cat conveys a wealth of binaural and spectral information when notch frequencies are identical. Type $\mathrm{O}$ units may enhance their spatial selectivity by encoding these directional cues in addition to notch frequency. Consequently, a more detailed analysis of type $\mathrm{O}$ responses to VSF stimuli may be expected to reveal the existence of directionally sensitive inputs that were not detected by our experiments with simple notched-noise analogs of the HRTF. For example, our studies of ITD coding in the inferior colliculus have identified a subset of type $\mathrm{O}$ units that are sensitive to binaural temporal cues that could be used to resolve the azimuth of isonotch HRTFs (Ramachandran and May 2002). Alternatively, the wideband integration properties of type $\mathrm{O}$ units may allow remote spectral features to modulate excitatory responses to the preferred notch frequency. Whatever their acoustic basis, these local processing effects appear to endow type $\mathrm{O}$ units with a special selectivity not only to spectral notches but also to the context in which they occur.

Delgutte et al. (1999) recently used VSF procedures to measure the spatial sensitivity of ICC neurons in the median plane. Approximately one-half of their units responded to simulated changes in sound source elevation. Some elevation-sensitive units showed spatially tuned rate peaks that are suggestive of the type $\mathrm{O}$ excitatory responses in Figure 9C. Other units displayed rate troughs that more likely reflect the type I inhibitory patterns in Figure 5D. Given this interpretation, only units encoding VSF locations with excitatory discharge rates would be expected to maintain their spatially selective responses if VSF stimuli were expanded to sound locations beyond the median plane.

Previous studies of the free-field spatial receptive fields of ICC neurons also have found populations of directionally sensitive units (Aitkin and Martin 1987, 1990). Many of the units in these studies displayed spatial tuning for both azimuth and elevation, with preferred locations concentrated at contralateral azimuths between $0^{\circ}$ and $50^{\circ}$ and elevations between $0^{\circ}$ and $40^{\circ}$. This range of spatial sensitivity corresponds well with the cat's optimum sound orientation re-
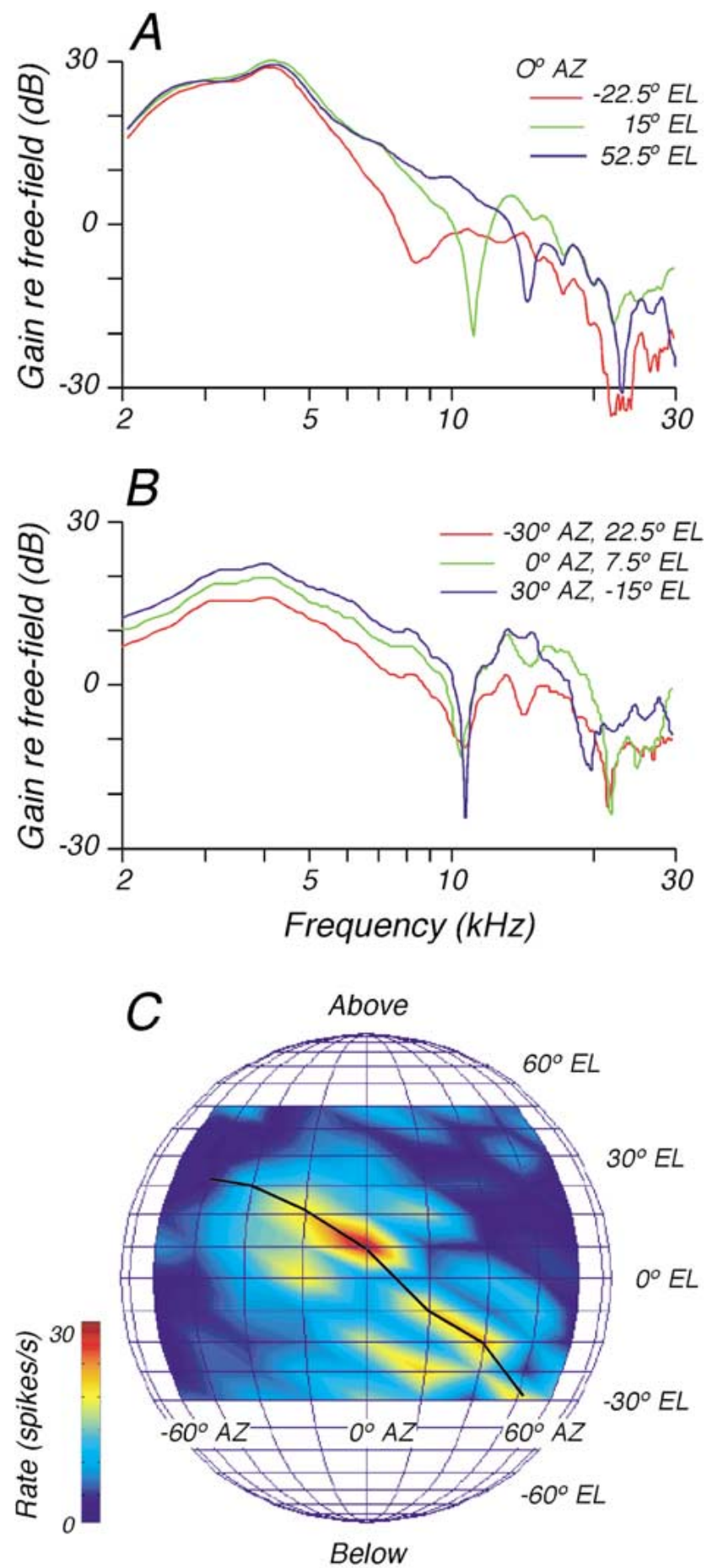

FIG. 9. Sensitivity of a type $O$ unit to directional properties of the cat's head-related transfer function. A. HRTFs for three elevations (ELs) at $0^{\circ}$ azimuth (AZ). The frequency of the large spectral notch between 8 and $15 \mathrm{kHz}$ is related to the direction of the sound source in the median plane. B. HRTFs for three locations thatproduce the same $11-\mathrm{kHz}$ notch frequency. $\mathbf{C}$. Spatial receptive field for a type $\mathrm{O}$ unit (2.04 exp. 9/9/99, BF = $12.6 \mathrm{kHz}$ ). HRTF filter shapes were used to synthesize binaural "virtual space" stimuli at azimuths and elevations where gridlines intersect. The unit achieved its highest discharge rates along the spatial contour where midfrequency notches fell near $11 \mathrm{kHz}$ (bold line). 
sponses (May and Huang 1996). Modeling studies based on the auditory nerve representation of spectral cues for sound localization predict orientation errors at more eccentric locations because midfrequency notches are not as prominent (May and $\mathrm{Hu}-$ ang 1997). Units with the best directional sensitivity in the studies of Aitkin and Martin typically had BFs in excess of $8 \mathrm{kHz}$, which closely matches the lowfrequency cutoff of spectral notches in the cat's HRTF (Rice et al. 1992).

Single-unit recordings in the medial geniculate body (MGB) of anesthetized cats suggest that type $\mathrm{O}$ outputs remain functionally segregated when they exit the inferior colliculus (Imig et al. 1997). A thalamic expression of the DCN/ICC spectral processing pathway is implied by a subclass of neurons with closed excitatory tuning curves and monaural directional (MD) sensitivity. MD units respond to broadband sounds with spatially tuned excitatory responses and therefore appear to derive their directionality from HRTF-based spectral cues. This spatial tuning can be shifted in azimuth or elevation by changing the orientation of the cat's pinna. DCN type IV units receive somatosensory inputs from the head and pinna and are similarly affected by pinna movements (Young et al. 1995, 1996). Our present characterization of type $\mathrm{O}$ units provides a solid foundation for investigating how the auditory processing of spectral information in the DCN and inferior colliculus contributes to spatial receptive fields in the thalamus (Imig et al. 1997) and auditory cortex (Samson et al. 1993).

\section{Generalization of type $\mathrm{O}$ response patterns to other studies and species}

Many studies have investigated single-unit responses in the ICC of cats and other species. Most of these previous recording paradigms did not include the frequency response maps upon which our system of physiological classification is based. Nevertheless, type $\mathrm{O}$ response patterns can be inferred from ratelevel functions for BF tones. This widely used physiological measure can be thought of as a frequencyspecific slice through the response map.

Relative to type $\mathrm{V}$ and type I units (Figs. 5A and B), only type $\mathrm{O}$ units show excitatory responses for $\mathrm{BF}$ tones at low sound levels and inhibitory responses at high levels (Figs. 1B and 6A). Such nonmonotonic or "closed" rate-level functions are a common attribute of the inferior colliculus and have been described in a variety of species including mice (Willott et al. 1988), gerbils (Semple and Kitzes 1987), guinea pigs (Rees and Palmer 1988), cats (Rose et al. 1963), bats (Pollak and Park 1993), and nonhuman primates (Ryan and Miller 1978).
The prevalence of nonmonotonic (type O-like) units has shown considerable variation in previous studies of the ICC. These differences may be amplified by simple procedural factors like the criteria that were used to distinguish between monotonic and nonmonotonic units, or by more complex physiological factors like the potential confounds of comparisons between anesthetized and awake subjects (for a review, see Irvine 1986). A more intriguing interpretation is that the distribution of type $\mathrm{O}$ units may reflect species-specific variations in the auditory processing of spectral information.

Recent studies of the inferior colliculus in anesthetized guinea pigs (LeBeau et al. 2001) have reported frequency response maps that follow the basic classification system of Ramachandran et al. (1999). Important species differences also were noted. Unlike the cat, units with $\mathrm{V}$-shaped response maps were observed at all audible frequencies and comprised the most common response pattern in guinea pigs. A less common non- $V$-shaped response class included units with narrowly tuned and closed receptive fields that are reminiscent of the type $\mathrm{I}$ and $\mathrm{O}$ units that are most frequently encountered in decerebrate cats. In addition to the rarity of type $\mathrm{O}$ responses in guinea pigs, the majority of these non- $\mathrm{V}$ units appeared to be created by local GABAergic inputs and could be transformed into type $\mathrm{V}$ responses by application of bicuculline.

Rodent species and cats also show important differences in their DCN response patterns (Davis et al. 1996). As in the ICC of anesthetized guinea pigs, closed frequency response maps are relatively rare in the DCN of decerebrate gerbils. Since these type IV responses are assumed to represent the precursor to low-rate type $\mathrm{O}$ units, a decrease in the availability of these inputs is expected to reduce the number of ICC units that display nonmonotonic rate-level functions or other more specific type $\mathrm{O}$ attributes.

Species differences in DCN and ICC physiology imply that ethological factors have shaped the functional expression of spectral processing pathways in the central auditory system. These specializations may be inferred by examining sound localization behavior in the context of predator/prey relationships (Heffner and Heffner 1987). Psychophysical studies have noted excellent directional acuity in predator species like the cat (Casseday and Neff 1973; Huang and May 1996b), but less accurate sound localization abilities in prey species like the guinea pig and gerbil (Clements and Kelly 1978; Kelly and Potash 1986; Heffner and Heffner 1988a). The influence of lifestyle has been used to explain the exceptional directional hearing of predatory rodents like the grasshopper mouse (Heffner and Heffner 1988b). This neurobehavioral perspective may be equally important in 
physiological descriptions of the general response patterns of the inferior colliculus and subsequent interpretations of the information processing roles of species-specific unit types (Masterton 1997).

\section{ACKNOWLEDGMENTS}

E. Young contributed archival data from his previous studies of the dorsal cochlear nucleus. This work was supported by National Institute of Deafness and Other Communication Disorders grant 5R01DC00954. Pharmacological manipulations were performed in conjunction with NIDCD grant 1R03DC03758.

\section{REFERENCES}

Aitkin LM, Martin RL. The representation of stimulus azimuth by high best-frequency azimuth-selective neurons in the central nucleus of the inferior colliculus of the cat. J. Neurophysiol. 57:1185-1200, 1987.

AitKIn LM, Martin RL. Neurons in the inferior colliculus of cats sensitive to sound-source elevation. Hear. Res. 50:97-105, 1990.

Aitkin LM, Webster WR, Veale JL, Crosby DC. Inferior colliculus. I. Comparison of response properties of neurons in central, pericentral, and external nuclei of adult cat. J. Neurophysiol. 38:1195-1207, 1975.

Batra R, Kuwada S, Fitzpatrick DC. Sensitivity to interaural temporal disparities of low- and high-frequency neurons in the superior olivary complex. I. Heterogeneity of responses. J. Neurophysiol. 78:1222-1236, 1997.

Cant NB, Casseday JH. Projections from the anteroventral cochlear nucleus to the lateral and medial superior olivary nuclei. J. Comp. Neurol. 247:457-476, 1986.

Casseday JH, Neff WD. Localization of pure tones. J. Acoust. Soc. Am. 54:365-372, 1973.

Clements M, Kelly JB. Auditory spatial responses of young guinea pigs (Cavia porcellus) during and after ear plugging. J. Comp. Physiol. Psychol. 92:34-44, 1978.

DAvIS KA. Evidence of a functionally segregated pathway from dorsal cochlear nucleus to inferior colliculus. J. Neurophysiol. 87:1824-1835, 2002.

Davis KA, Ding J, Benson TE, Voigt HF . Response properties of units in the dorsal cochlear nucleus of unanesthetized decerberate gerbil. J. Neurophysiol. 75:1411-1431, 1996.

Davis KA, Ramachandran R, May BJ. Single-unit responses in the inferior colliculus of decerebrate cats. II. Sensitivity to interaural level differences. J. Neurophysiol. 82:164-175, 1999.

Delgutte B, Joris PX, Litovsky RY, Yin TC. Receptive fields and binaural interactions for virtual-space stimuli in the cat inferior colliculus. J. Neurophysiol. 81:2833-2851, 1999.

Havey DC, Caspary DM. A simple technique for constructing 'piggy-back' multibarrel microelectrodes. Electroenceph. Clin. Neurophysiol. 48:249-251, 1980.

Heffner RS, Heffner HE. Localization of noise, use of binaural cues, and a description of the superior olivary complex in the smallest carnivore, the least weasel (Mustel nivalis). Behav. Neurosci. 101:701-708, 1987.

Heffner RS, Heffner HE. Sound localization and use of binaural cues by the gerbil (Mustel nivalis). Behav. Neurosci. 102:422428, 1988a.

Heffner RS, Heffner HE. Sound localization in a predatory rodent, the northern grasshopper mouse (Onychomys leucogaster). J. Comp. Psychol. 102:66-71, 1988b.
HuAng AY, MAY BJ. Sound orientation behavior in cats. II. Midfrequency spectral cues for sound localization. J. Acoust. Soc. Am. 100:1070-1080, 1996a.

HuANG AY, MAY BJ. Spectral cues for sound localization in cats. Effects of frequency domain on minimum audible angles in the median and horizontal planes. J. Acoust. Soc. Am. 100:23412348, 1996b.

Imig TJ, Bibikov NG, Poirier P, SAmson FK. Directionality derived from pinna-cue spectral notches in cat dorsal cochlear nucleus. J. Neurophysiol. 83:907-925, 2000.

Imig TJ, Poirier P, Irons WA, SAmson FK. Monaural spectral contrast mechanism for neural sensitivity to sound direction in the medial geniculate body of the cat. J. Neurophysiol. 78:27542771, 1997.

IRVINE DRF. The Auditory Brainstem. In: Autrum H, Ottoson D, Perl ER, Schmidt RF, Shimazu H, Willis D (eds) Progress in Sensory Physiology, Vol. 7 Springer-Verlag, Berlin, 1986, pp 128-211.

Kelly JB, Potash M. Directional responses to sounds in young gerbils (Meriones unguiculatus). J. Comp. Psychol. 100:37-45, 1986.

LeBeau FE, Malmierca MS, Rees A. Iontophoresis in vivo demonstrates a key role for $\mathrm{GABA}(\mathrm{A})$ and glycinergic inhibition in shaping frequency response areas in the inferior colliculus of guinea pig. J. Neurosci. 21:7303-7312, 2001.

MAY BJ. Role of the dorsal cochlear nucleus in the sound localization behavior of cats. Hear. Res. 148:74-87, 2000.

MaY BJ, Huang AY. Sound orientation behavior in cats: I. Localization of broadband noise. J. Acoust. Soc. Am. 100:1059-1069, 1996.

May BJ, Huang AY. Spectral cues for sound localization in cats: A model for discharge rate representations in the auditory nerve. J. Acoust. Soc. Am. 101:2705-2719, 1997.

MASTERTON RB. Neurobehavioral studies of the central auditory system. Ann. Otol. Rhinol. Laryngol. Suppl 168:31-34, 1997.

Merzenich MM, ReID MD. Representation of the cochlea within the inferior colliculus of the cat. Brain. Res. 77:397-415, 1974.

Musicant AD, Chan JC, Hind JE. Direction-dependent spectral properties of cat external ear: new data and cross-species comparisons. J. Acoust. Soc. Am. 87:757-781, 1990.

Nelken I, Young ED. Linear and nonlinear spectral integration in type IV neurons of the dorsal cochlear nucleus. I. Regions of linear interaction. J. Neurophysiol. 78:800-811, 1997.

Nelken I, Young ED. Two separate inhibitory mechanisms shape the responses of dorsal cochlear nucleus type IV neurons to narrowband and wideband stimuli. J. Neurophysiol. 71:24462462, 1994.

Oliver DL, Beckius G. Fine structure of GABA-labeled axonal endings in the inferior colliculus of the cat. Immunocytochemistry on deplasticized ultrathin sections. Neuroscience 46:455-463, 1992.

Oliver DL, Beckius GE, Bishop DC, Kuwada S. Simultaneous anterograde labeling of axonal layers from lateral superior olive and dorsal cochlear nucleus in the inferior colliculus of cat. J. Comp. Neurol. 382:215-229, 1997.

Pollak GD, PARK TJ. The effects of GABAergic inhibition on monaural response properties of neurons in the mustache bat's inferior colliculus. Hear. Res. 65:99-117, 1993.

PoOn PWF, BRugge JF. Sensitivity of auditory nerve fibers to spectral notches. J. Neurophysiol. 70:655-666, 1993.

Ramachandran R, Davis KA, May BJ. Single-unit responses in the inferior colliculus of decerebrate cats. I. Classification based on frequency response maps. J. Neurophysiol. 82:152-163, 1999.

Ramachandran R, Davis KA, May BJ. Rate representation of tones in noise in the inferior colliculus of decerebrate cats. J. Assoc. Res. Otolaryngol. 1:144-160, 2000. 
RAMACHANDRAN R, MAY BJ. Sensitivity to interaural time differences in the inferior colliculus of decerberate cats. J. Neurophysiol., in press.

Rees A, Palmer AR. Rate-intensity functions and their modification by broadband noise for neurons in the guinea pig inferior colliculus. J. Acoust. Soc. Am. 83:1488-1498, 1988.

Rice JJ, May BJ, Spirou GA, Young ED. Pinna based spectral cues for sound localization in cat. Hear. Res. 58:132-152, 1992.

Rose JE, Greenwood DD, Goldberg JM, Hind JE. Some discharge characteristics of single neurons in the inferior colliculus of the cat. I. Tonotopical organization, relation of spike-counts to tone intensity, and firing patterns of single elements. J. Neurophysiol. 26:294-320, 1963.

Ryan AF, Miller J. Single unit responses in the inferior colliculus of the awake and performing rhesus monkey. Exp. Brain Res. 32:389-407, 1978.

Ryugo DK, Willard FH, Fekete DM. Differential afferent projections to the inferior colliculus from the cochlear nucleus in the albino mouse. Brain Res. 210:342-349, 1981.

Samson FK, Clarey JC, Barone P, Imig TJ. Effects of ear plugging on single-unit azimuth sensitivity in cat primary auditory cortex. I. Evidence for monaural directional cues. J. Neurophysiol. 70:492-511, 1993.

SEMPle MN, Kitzes LM. Binaural processing of sound pressure level in the inferior colliculus. J. Neurophysiol 57:1130-1147, 1987.

Shneiderman A, Chase MB, Rockwood JM, Benson CG, Potashner SJ. Evidence for a GABAergic projection from the dorsal nu- cleus of the lateral lemniscus to the inferior colliculus. J. Neurochem. 60:72-82, 1993.

Shu ZJ, Swindale NV, Cynader MS. Spectral motion produces an auditory after-effect. Nature 364:721-723, 1993.

Spitzer MW, SEmple MN. Interaural phase coding in the auditory midbrain: Influence of dynamic stimulus features. Science 254:721-724, 1991.

Sutherland DP, Masterton RB, GLEndenning KK. Role of acoustic striae in hearing: reflexive responses to elevated sound-sources. Behav. Brain Res. 97:1-12, 1998.

Wightman FL, Kistler DJ. Headphone simulation of free-field listening. I. Stimulus synthesis. J. Acoust. Soc. Am. 85:868-878, 1989.

Willott JF, Parham K, Hunter KP. Response properties of inferior colliculus neurons in young and very old CBA/J mice. Hear. Res. 37:1-14, 1998.

Young ED, BRownell WE. Responses to tones and noise of single cells in the dorsal cochlear nucleus of unanesthetized cats. J. Neurophysiol. 39:282-300, 1979.

Young ED, Nelken I, Conley RA, Tong SC. Somatosensory effects on neurons in dorsal cochlear nucleus. J. Neurophysiol. 73:743$765,1995$.

Young ED, Rice JJ, Tong SC. Effects of pinna position on headrelated transfer functions in the cat. J. Acoust. Soc. Am. 99:3064-3076, 1996.

Young ED, Spirou GA, Rice JJ, Voigt HF. Neural organization and responses to complex stimuli in the dorsal cochlear nucleus. Philos. Trans. R. Soc. Lond. Biol. Sci. 336:407-413, 1992. 